\title{
Autism and cancer share risk genes, pathways and drug targets
}

\author{
Jacqueline N. Crawley ${ }^{1,2}$, Wolf-Dietrich Heyer ${ }^{3,4}$, Janine M. LaSalle ${ }^{1,4,5}$ \\ ${ }^{1}$ MIND Institute, ${ }^{2}$ Department of Psychiatry \& Behavioral Sciences, School of Medicine, \\ University of California Davis, Sacramento, CA 95817, USA \\ ${ }^{3}$ Department of Microbiology \& Molecular Genetics, University of California Davis, Davis, CA \\ 95616, USA \\ ${ }^{4}$ Comprehensive Cancer Center, University of California Davis, Sacramento, CA, 95817, USA \\ ${ }^{5}$ Department of Medical Microbiology \& Immunology and Genome Center \\ University of California Davis, Davis, CA 95616, USA
}

Submitted to Trends in Genetics as a Forum piece, October $26^{\text {th }}, 2015$

Revised December $18^{\text {th }}, 2015$

Keywords: Autism, cancer, gene, signaling pathway, chromatin remodeling, DNA repair

Corresponding Author:

Jacqueline N. Crawley, Ph.D.

Department of Psychiatry and Behavioral Sciences

Room 1001A Research II Building 96

4625 Second Avenue, Sacramento, CA 95817 USA

crawley@ucdavis.edu

1-916-734-1129

(C) 2016. This manuscript version is made available under the Elsevier user license 


\begin{abstract}
Autism is a neurodevelopmental disorder, diagnosed behaviorally by social and communication deficits, repetitive behaviors and restricted interests. Recent genome-wide exome sequencing has revealed extensive overlap in risk genes for autism and for cancer. Understanding the genetic commonalities of autism(s) and cancer(s), with a focus on mechanistic pathways, could lead to repurposed therapeutics.
\end{abstract}


Autism is a neurodevelopmental disorder, diagnosed by behavioral symptoms including impaired social interactions and communication, repetitive behaviors and restricted interests [1]. Extraordinarily high heritability for autism spectrum disorder (ASD) has been detected in twin studies, with a range of 50-90\% concordance between monozygotic twins, as compared to $0-30 \%$ between dizygotic twins and siblings, and approximately $1 \%$ prevalence in the general population, along with a high male:female ratio [2]. International consortia searching for the genetic causes of ASD quickly recognized that autism is not a monogenic disorder. Hundreds of de novo and familial risk genes, copy number variants and epigenetic modifiers have been identified through linkage analysis, genome wide-association studies, exon and whole genome sequencing of individuals with ASD over the last 2 years [2-5].

Table 1 summarizes the characteristics of risk genes for ASD that are also risk genes for cancers, extending the original finding that the PI3K-Akt-mTOR signaling axis (involving PTEN, FMR1, NF1, TSC1, TSC2) was associated with inherited risk for both cancer and ASD [6-9]. Recent genome-wide exome sequencing studies of de novo variants in ASD and cancer have begun to uncover considerable additional overlap. What is surprising about the genes in Table 1 is not necessarily the number of risk genes found in both autism and cancer, but the shared functions of genes in chromatin remodeling and genome maintenance, transcription factors, and signal transduction pathways leading to nuclear changes [7,8]. Chromatin remodeling factors important in altering nucleosome accessibility for transcription and genome maintenance mechanisms include $C H D 8, C H D 7, C H D 2, A R I D 1 B$, and ATRX. ATRX may exert a more specific function in telomere maintenance, analogous to other Swi2/Snf2 family factors such as ERCC6, RAD54, HTLF, SHPRH, or RAD16, which function in dedicated DNA repair 
pathways. Proteins involved in histone methyltransferase reactions important in setting the histone code include ASHL1, EHMT1, EHMT2, KMT2C, KMT2D, and SUV42OH1. PHF2, $K D M 5 B$, and $K D M 6 B$ are histone demethylases, and MACROD2 encodes a nuclear factor regulated by a metabolite of histone deacetylation. Ubiquitin modifications to histones and other proteins are implicated by the risk genes CUL3, HERC2, MIB1, TBL1XR1, TRIP12, UBE3A, and WAC. Transcription factors genetically implicated in both autism and cancer include ADNP, $P A X 5$, FOXP1, TCF7L2, and TBLXR1. Interestingly, these nuclear factors are downstream of several key signal transduction pathways also genetically implicated in ASD and cancer, including PTEN [7]. PTEN functions in the AKT signaling pathway, where its phosphatase activity is needed for AKT downregulation. Nuclear PTEN also regulates recombinational DNA repair, a key genome maintenance pathway (see below). It is unclear whether this is related to its signaling function or a consequence of a second independent PTEN activity, but this dual function may provide the rationale for the dominant role of PTEN in cancer and autism. Other genes encoding common tumor signaling pathways include MET (mitogen inducible gene 8), PTK7, and HRAS, while p53, AKT, mTOR, WNT, NOTCH, and MAPK are components of signaling pathways regulating the nuclear factors described above.

Autism is comorbid with several monogenic neurodevelopmental disorders including Fragile X (FMR1), Rett syndrome (MECP2), Phelan-McDermid (SHANK3), 15q duplication syndrome (UBE3A), neurofibromatosis (NF1), Tuberous sclerosis (TSC1, TSC2) and Cornelia de Lange syndrome (NIPBL, SMC1A) (Table 1). Neurofibromatosis and tuberous sclerosis are directly associated with tumors, but such tumors are benign and rarely if at all associated with malignancies. However, mutations in $N F 1, T S C 1$ or $T S C 2$ do enhance the risk for developing cancer [6]. Notably, NF1, TSC1 and TSC2 function like PTEN in the AKT pathway of mTOR 
control. Mutations in transcriptional factor genes also mediate downstream signaling pathways which include key proteins implicated in cell proliferation or differentiation pathways implicated in cancer and autism, such as mTOR, RAS GTPases, MAP kinases, AKT, EIF4E, WNT, ERK, PI3K, CHD8. A risk gene originally identified in individuals with cancer may present as a de novo mutation in a small number of individuals with ASD, or may be implicated in ASD through interactome analysis of interrelated genes and interacting proteins, e.g. within a signaling pathway (Table 1).

What does tumor cell proliferation have in common with brain development and neuronal synapse formation? Like cancers, "autisms" are best conceptualized in the plural. ASD encompasses a broad range of putative causes, symptom presentations, and outcomes, including both macrocephaly and microcephaly, suggesting deficits in the cellular commitment to proliferation versus differentiation, similar to cancer. This difference may be in the life stage of cellular proliferation. Errors associated with genome maintenance during fetal life may occur at critical time periods for proliferation of neuronal precursors that affect prenatal brain development, resulting in neurodevelopmental disorders, whereas errors more commonly occur during adult life in cell types susceptible to tumors. Biological mechanisms with potential commonalities between genes implicated in both cancers and autisms may be revealed from a closer investigation of the specific actions of genes and converging pathways identified in both [8]. For example, UBE3A, which is duplicated in $~ 1-2 \%$ of ASD, encodes the ubiquitin E3 ligase protein E6-AP, first named as an E6 interacting protein that degrades p53 in human cervical cancer [10].

The intersection between autism and cancer in genome maintenance pathways is novel and particularly compelling. A large cohort of autism and cancer genes affect genome 
maintenance including signaling molecules (PTEN), DNA repair factors (ERCC6, SMARCA2), structural chromosome components such as cohesins (NIPBL, SMC1A, SMC2), factors needed for Alternative Lengthening of Telomeres (ATRX), and post-translational modifiers (TRIP12, $U B E 3 A, H E R C 2)$. The functional overlap goes beyond this common gene set, as genomes from individuals with ASD show mutational hotspots and a high incidence of copy number variations. These genetic events signal pathological outcomes of DNA replication stress. Many neuronspecific genes are rather large with primary transcripts in the Mbp range. Such genes are at particular risk for transcription-DNA replication conflicts that underpin a significant amount of genome instability [11]. While these genes are typically transcribed only in terminally differentiated cells, any miscoordination of transcriptional control, DNA replication, differentiation, and cell cycle phasing will greatly increase the risk of mutations targeted to these genes encoding critical brain functions. Transcription-coupled repair, the pathway defined by ERCC6, is of particular importance for terminally differentiated cells and long transcription units. Overall too little is known about DNA repair in terminally differentiated cells and more studies are needed to evaluate other pathways such as recombinational DNA repair in differentiated cells and somatic genomic instability in neurons. Thus, similar to cancer, the inherited risk for autism may be compounded by further somatic mutations associated with mutations in known risk genes that may be biased for genes with neuronal functions.

The functional overlap of genes and pathways between autism and cancer would suggest that individuals with autism may carry a higher cancer risk. While there is some epidemiological evidence of higher cancer risk in children, adolescents, and young adults with ASD [9, 12], the absolute number of cases is low and more studies need to be conducted, particularly in adults, as cancer incidence is significantly correlated with age. 
Mouse models with mutations in many of these genes have been widely used in both cancer and autism research. Some of these mutant mouse models recapitulate behavioral and biological features of autism [13]. These model systems are proving useful in understanding the consequences of specific mutations on overgrowth of brain regions, unusual patterns of white matter connectivity, aberrant numbers of synapses, and altered morphology of dendritic spines, in parallel to understanding cell proliferation, cell cycle, DNA repair, and epigenetic causes in malignancies.

Considerable translational value can be gained from a new focus to understand the genetic commonalities of autism(s) and cancer(s). Importantly, mechanistic similarities can be leveraged into therapeutic strategies. It may be possible to repurpose available cancer drugs with reasonable safety profiles as targeted treatments for ASD. For example, evaluation of a rapamycin analogue in tuberous sclerosis patients included outcome measures for ASD features, along with seizures, sleep disturbances and academic skills (NCT01289912, ClinicalTrials.gov). Stratifying individuals with ASD who harbor a risk gene for autism that is also a risk gene for cancer may enable therapeutic development of personalized medicines based on the specific causal mutation.

Table 1. Characteristics of risk genes implicated in both autism and cancer

\begin{tabular}{|c|c|c|c|c|c|c|c|}
\hline $\begin{array}{l}\text { Gene } \\
\text { name }\end{array}$ & aliases & $\begin{array}{l}\text { Huma } \\
\mathbf{n} \\
\text { chrom } \\
\text { locatio } \\
\mathrm{n}\end{array}$ & $\begin{array}{l}\text { Protein } \\
\text { function }\end{array}$ & $\begin{array}{l}\text { Interacting } \\
\text { proteins }\end{array}$ & $\begin{array}{l}\text { Autism related } \\
\text { neurodevelopm } \\
\text { ental syndrome }\end{array}$ & $\begin{array}{l}\text { Cancer } \\
\text { susceptibilit } \\
\text { y or } \\
\text { pathway }\end{array}$ & $\begin{array}{l}\text { Refs } \\
\text { (PMID) }\end{array}$ \\
\hline$A D N P$ & $\begin{array}{l}\text { Activity- } \\
\text { dependent } \\
\text { neuroprotecto } \\
\text { r homeobox }\end{array}$ & $\begin{array}{l}20 q 13 . \\
13\end{array}$ & $\begin{array}{l}\text { Potential } \\
\text { transcription } \\
\text { factor. May } \\
\text { mediate some } \\
\text { of the } \\
\text { neuroprotecti } \\
\text { ve peptide }\end{array}$ & $\begin{array}{l}\text { SMARCA4, } \\
\text { SMARCC2, } \\
\text { ARID1A }\end{array}$ & $\begin{array}{l}\text { Helsmoortel- } \\
\text { van der } \mathrm{Aa} \\
\text { syndrome }\end{array}$ & p53, WNT & $\begin{array}{l}25891 \\
009\end{array}$ \\
\hline
\end{tabular}




\begin{tabular}{|c|c|c|c|c|c|c|c|}
\hline & & & $\begin{array}{l}\text { VIP-associated } \\
\text { effects }\end{array}$ & & & & \\
\hline ANK2 & $\begin{array}{l}\text { Ankyrin 2, } \\
\text { Neuronal }\end{array}$ & $4 q 25$ & $\begin{array}{l}\text { Attaches } \\
\text { integral } \\
\text { membrane } \\
\text { proteins to } \\
\text { cytoskeletal } \\
\text { elements and } \\
\text { regulates cell } \\
\text { motility, } \\
\text { activation, } \\
\text { proliferation, } \\
\text { and contact }\end{array}$ & $\begin{array}{l}\text { DMD, } \\
\text { DCTN4, } \\
\text { ACTF1 }\end{array}$ & $\begin{array}{l}\text { Long } \\
\text { (Electrocardiogr } \\
\text { aphic) QT } \\
\text { Syndrome } 4\end{array}$ & $\begin{array}{l}\text { proteoglyca } \\
\text { ns }\end{array}$ & $\begin{array}{l}25863 \\
124\end{array}$ \\
\hline$A R I D 1 B$ & $\begin{array}{l}\text { AT Rich } \\
\text { Interacting } \\
\text { Domain 1B } \\
\text { (SWI1-like), } \\
\text { BRG1-Binding } \\
\text { protein }\end{array}$ & $6 q 25.3$ & $\begin{array}{l}\text { Subunit of } \\
\text { SWI/SNF } \\
\text { chromatin } \\
\text { remodelimg } \\
\text { complex }\end{array}$ & $\begin{array}{l}\text { ARID1A, } \\
\text { SMARCA2, } \\
\text { RELB, } \\
\text { SMAD9, } \\
\text { ASF1A }\end{array}$ & $\begin{array}{l}\text { Coffin-Siris } \\
\text { syndrome }\end{array}$ & $\begin{array}{l}\text { ESR1, WNT; } \\
\text { prostate } \\
\text { cancer }\end{array}$ & $\begin{array}{l}25891 \\
009\end{array}$ \\
\hline$A S H 1 L$ & $\begin{array}{l}\text { Lysine } \mathrm{N}- \\
\text { Methyltransfer } \\
\text { ase } 2 \mathrm{H}\end{array}$ & $1 q 22$ & $\begin{array}{l}\text { Histone } \\
\text { methyltransfer } \\
\text { ase specifically } \\
\text { methylating } \\
\text { Lys-36 of } \\
\text { histone H3 } \\
\text { (H3K36me) }\end{array}$ & $\begin{array}{l}\text { SMAD7, } \\
\text { HIST1H3A }\end{array}$ & $\begin{array}{l}\text { Autism, } \\
\text { susceptibility }\end{array}$ & $\begin{array}{l}\text { Lysine } \\
\text { degradation }\end{array}$ & $\begin{array}{l}26402 \\
605\end{array}$ \\
\hline ATRX & $\begin{array}{l}\text { RAD54, Alpha } \\
\text { Thalassemia/ } \\
\text { Mental } \\
\text { Retardation } \\
\text { Syndrome X- } \\
\text { linked }\end{array}$ & Xq21.1 & $\begin{array}{l}\text { SWI/SNF ATP- } \\
\text { dependent } \\
\text { DNA motor } \\
\text { protein that } \\
\text { acts in } \\
\text { heterochroma } \\
\text { tin and } \\
\text { telomere }\end{array}$ & $\begin{array}{l}\text { CBX5, DAXX, } \\
\text { HDAC1, } \\
\text { SMC1A, } \\
\text { SMC3 }\end{array}$ & $\begin{array}{l}\text { Alpha- } \\
\text { thalassemia/me } \\
\text { ntal retardation } \\
\text { syndrome }\end{array}$ & $\begin{array}{l}\text { breast } \\
\text { cancer, } \\
\text { telomeres }\end{array}$ & $\begin{array}{l}24779 \\
060\end{array}$ \\
\hline CHD2 & $\begin{array}{l}\text { Chromodomai } \\
\mathrm{n} \text { Helicase DNA } \\
\text { Binding } \\
\text { Protein 2, ATP- } \\
\text { dependent } \\
\text { helicase }\end{array}$ & $\begin{array}{l}15 q 26 . \\
1\end{array}$ & $\begin{array}{l}\text { SWI/SNF ATP- } \\
\text { dependent } \\
\text { DNA motor } \\
\text { protein that } \\
\text { acts as a } \\
\text { chromatin } \\
\text { remodeling } \\
\text { factor and } \\
\text { transcriptional } \\
\text { regulator, also } \\
\text { DNA repair }\end{array}$ & $\begin{array}{l}\text { SUMO1, } \\
\text { PARK7 }\end{array}$ & $\begin{array}{l}\text { Epileptic } \\
\text { encephalopathy } \\
\text {, childhood- } \\
\text { onset }\end{array}$ & $\begin{array}{l}\text { Chromatin } \\
\text { regulation }\end{array}$ & $\begin{array}{l}25891 \\
009\end{array}$ \\
\hline
\end{tabular}




\begin{tabular}{|c|c|c|c|c|c|c|c|}
\hline CHD7 & $\begin{array}{l}\text { Chromodomai } \\
\mathrm{n} \text { Helicase DNA } \\
\text { Binding } \\
\text { Protein 7, ATP- } \\
\text { dependent } \\
\text { helicase }\end{array}$ & $8 q 12.2$ & $\begin{array}{l}\text { SWI/SNF ATP- } \\
\text { dependent } \\
\text { DNA motor } \\
\text { protein that } \\
\text { acts as a } \\
\text { chromatin } \\
\text { remodeling } \\
\text { factor and } \\
\text { transcriptional } \\
\text { regulator }\end{array}$ & $\begin{array}{l}\text { CHD8, } \\
\text { PBRM1, } \\
\text { SMARCC1, } \\
\text { SMARCC2, } \\
\text { SMARCE1 }\end{array}$ & $\begin{array}{l}\text { CHARGE } \\
\text { syndrome }\end{array}$ & $\begin{array}{l}\text { WNT } \\
\text { signalling, } \\
\text { chromatin } \\
\text { regulation }\end{array}$ & $\begin{array}{l}24768 \\
552\end{array}$ \\
\hline CHD8 & $\begin{array}{l}\text { Chromodomai } \\
\text { n Helicase DNA } \\
\text { Binding } \\
\text { Protein 8, } \\
\text { HELSNF1, } \\
\text { AUTS18 }\end{array}$ & $\begin{array}{l}14 q 11 . \\
2\end{array}$ & $\begin{array}{l}\text { SWI/SNF ATP- } \\
\text { dependent } \\
\text { DNA motor } \\
\text { protein that } \\
\text { acts as a } \\
\text { chromatin } \\
\text { remodeling } \\
\text { factor and } \\
\text { transcriptional } \\
\text { regulator }\end{array}$ & $\begin{array}{l}\text { RBBP5, } \\
\text { WDR5, } \\
\text { CTNNB1, } \\
\text { USF1, CTCF }\end{array}$ & $\begin{array}{l}\text { Autism, } \\
\text { susceptibility }\end{array}$ & $\begin{array}{l}\text { WNT } \\
\text { signalling, } \\
\text { chromatin } \\
\text { regulation }\end{array}$ & $\begin{array}{l}25891 \\
009\end{array}$ \\
\hline CUL3 & Cullin 3 & $2 q 36.2$ & $\begin{array}{l}\text { Core } \\
\text { component of } \\
\text { multiple cullin- } \\
\text { RING-based } \\
\text { BCR (BTB- } \\
\text { CUL3-RBX1) E3 } \\
\text { ubiquitin- } \\
\text { protein ligase } \\
\text { complex }\end{array}$ & $\begin{array}{l}\text { KLHL3, } \\
\text { NEDD8, } \\
\text { KEAP1, RBX1, } \\
\text { CASP8 }\end{array}$ & $\begin{array}{l}\text { Autism, } \\
\text { susceptibility }\end{array}$ & $\begin{array}{l}\text { WNT } \\
\text { signalling, } \\
\text { chromatin } \\
\text { regulation }\end{array}$ & $\begin{array}{l}25363 \\
768\end{array}$ \\
\hline $\begin{array}{l}\text { DNMT3 } \\
A\end{array}$ & $\begin{array}{l}\text { DNA (5- } \\
\text { cytosine)- } \\
\text { methyltransfer } \\
\text { ase } 3 \mathrm{~A}\end{array}$ & $2 p 23.3$ & $\begin{array}{l}\text { Required for } \\
\text { genome-wide } \\
\text { de novo } \\
\text { methylation } \\
\text { and is } \\
\text { essential for } \\
\text { the } \\
\text { establishment } \\
\text { of DNA } \\
\text { methylation } \\
\text { patterns } \\
\text { during } \\
\text { development }\end{array}$ & $\begin{array}{l}\text { DNMT3L, } \\
\text { DNMT3B, } \\
\text { UHRF1 }\end{array}$ & $\begin{array}{l}\text { Autism, } \\
\text { susceptibility }\end{array}$ & $\begin{array}{l}\text { Chromatin } \\
\text { regulation }\end{array}$ & $\begin{array}{l}26402 \\
605\end{array}$ \\
\hline $\begin{array}{l}\text { DYRK1 } \\
\text { A }\end{array}$ & $\begin{array}{l}\text { Dual-specificity } \\
\text { tyrosine } \\
\text { phosphorylatio } \\
\text { n-regulated } \\
\text { kinase } 1 \mathrm{~A}\end{array}$ & $\begin{array}{l}21 q 22 . \\
13\end{array}$ & $\begin{array}{l}\text { serine/threoni } \\
\text { ne kinase } \\
\text { implicated in } \\
\text { cell survival, } \\
\text { proliferation } \\
\text { and }\end{array}$ & $\begin{array}{l}\text { HIPK2, SFN, } \\
\text { YWHAB, } \\
\text { YWHAE, } \\
\text { DCAF }\end{array}$ & $\begin{array}{l}\text { Down } \\
\text { syndrome, } \\
\text { mental } \\
\text { retardation, } \\
\text { autosomal } \\
\text { dominant } 7\end{array}$ & $\begin{array}{l}\text { NOTCH } \\
\text { signalling, } \\
\text { translation } \\
\text { regulation }\end{array}$ & $\begin{array}{l}17583 \\
556\end{array}$ \\
\hline
\end{tabular}




\begin{tabular}{|c|c|c|c|c|c|c|c|}
\hline & & & differentiation & & & & \\
\hline EHMT1 & $\begin{array}{l}\text { Euchromatic } \\
\text { Histone-Lysine } \\
\mathrm{N} \text { - } \\
\text { Methyltransfer } \\
\text { ase, KMT1D, } \\
\text { CLP }\end{array}$ & $9 q 34.3$ & $\begin{array}{l}\text { Histone } \\
\text { methyltransfer } \\
\text { ase of } \\
\text { H3K9me and } \\
\text { H3K9me2 in } \\
\text { euchromatin }\end{array}$ & $\begin{array}{l}\text { MDM2, p53, } \\
\text { SUV39H1, } \\
\text { HIST1H3A, } \\
\text { CTBP1, } \\
\text { SUV39H1 }\end{array}$ & $\begin{array}{l}\text { Kleefstra } \\
\text { syndrome }\end{array}$ & $\begin{array}{l}\text { cellular } \\
\text { senescence, } \\
\text { NOTCH, } \\
\text { lysine } \\
\text { degradation }\end{array}$ & $\begin{array}{l}24779 \\
060\end{array}$ \\
\hline $\begin{array}{l}E R B B 2 I \\
P\end{array}$ & $\begin{array}{l}\text { ERBB2 } \\
\text { Interacting } \\
\text { protein }\end{array}$ & $5 q 12.3$ & $\begin{array}{l}\text { Acts as an } \\
\text { adapter for } \\
\text { the receptor } \\
\text { ERBB2, } \\
\text { inhibits NOD2- } \\
\text { dependent } \\
\text { NF-kappa-B } \\
\text { signaling and } \\
\text { proinflammat } \\
\text { ory cytokine } \\
\text { secretion }\end{array}$ & $\begin{array}{l}\text { ERBB2, } \\
\text { SMAD2, } \\
\text { SMAD3, } \\
\text { NRG2, PKP4 }\end{array}$ & $\begin{array}{l}\text { Autism, } \\
\text { susceptibility }\end{array}$ & $\begin{array}{l}\text { TGFb } \\
\text { signalling, } \\
\text { cervical and } \\
\text { colon } \\
\text { cancer }\end{array}$ & $\begin{array}{l}26402 \\
605\end{array}$ \\
\hline ERCC6 & $\begin{array}{l}\text { Cockayne's } \\
\text { Syndrome B }\end{array}$ & $\begin{array}{l}10 q 11 . \\
23\end{array}$ & $\begin{array}{l}\text { SWI/SNF ATP- } \\
\text { dependent } \\
\text { DNA motor } \\
\text { protein that } \\
\text { acts in } \\
\text { transcription- } \\
\text { coupled DNA } \\
\text { repair }\end{array}$ & $\begin{array}{l}\text { Cockayne's } \\
\text { Syndrome- } \\
\text { A/ERCC8 } \\
\text { TFIIH, } \\
\text { SMARCA5/S } \\
\text { NF2H, } \\
\text { BAZ1B/WSTF } \\
\text {, SF3B1, DEK, } \\
\text { MYO1C, } \\
\text { MYBBP1A, } \\
\text { DDX21, } \\
\text { KIAA1530/U } \\
\text { VSSA. }\end{array}$ & $\begin{array}{l}\text { High confidence } \\
\text { ASD candidate } \\
\text { gene }\end{array}$ & $\begin{array}{l}\text { transcriptio } \\
\text { n-coupled } \\
\text { DNA repair }\end{array}$ & $\begin{array}{l}24768 \\
552\end{array}$ \\
\hline FOXP1 & $\begin{array}{l}\text { Forkhead box } \\
\text { P1 }\end{array}$ & $3 p 13$ & $\begin{array}{l}\text { Forkhead box } \\
\text { transcription } \\
\text { factor and } \\
\text { putative } \\
\text { tumor } \\
\text { suppressor }\end{array}$ & $\begin{array}{l}\text { CTBP1, } \\
\text { FOXP2, } \\
\text { FOXP4, MYC, } \\
\text { NCOR2 }\end{array}$ & $\begin{array}{l}\text { Autism, } \\
\text { susceptibility }\end{array}$ & $\begin{array}{l}\text { WNT, Notch } \\
\text { signaling }\end{array}$ & $\begin{array}{l}25363 \\
768\end{array}$ \\
\hline HERC2 & $\begin{array}{l}\text { HECT And RLD } \\
\text { Domain } \\
\text { Containing E3 } \\
\text { Ubiquitin } \\
\text { Protein Ligase } \\
2\end{array}$ & $15 q 13$ & $\begin{array}{l}\text { E3 ubiquitin- } \\
\text { protein ligase } \\
\text { that regulates } \\
\text { repair proteins } \\
\text { on damaged } \\
\text { chromosomes, } \\
\text { regulates } \\
\text { replication } \\
\text { fork }\end{array}$ & $\begin{array}{l}\text { UBE3A, } \\
\text { SUMO1, } \\
\text { RNF8, BRCA1 }\end{array}$ & $\begin{array}{l}\text { Mental } \\
\text { retardation, } \\
\text { autosomal } \\
\text { recessive } 38 \\
\text { (MRT38) }\end{array}$ & $\begin{array}{l}\text { Class I MHC } \\
\mathrm{Ag} \\
\text { presentatio } \\
\mathrm{n} \text { and } \\
\text { processing }\end{array}$ & $\begin{array}{l}24779 \\
060\end{array}$ \\
\hline
\end{tabular}




\begin{tabular}{|c|c|c|c|c|c|c|c|}
\hline & & & progression & & & & \\
\hline HRAS & $\begin{array}{l}\text { Harvey Rat } \\
\text { Sarcoma Viral } \\
\text { Oncogene } \\
\text { Homolog, } \\
\text { p21RAS }\end{array}$ & $\begin{array}{l}11 \mathrm{p} 15 . \\
5\end{array}$ & $\begin{array}{l}\text { RAS oncogene } \\
\text { family } \\
\text { members that } \\
\text { bind GTP and } \\
\text { GDP, with } \\
\text { intrinsic } \\
\text { GTPase } \\
\text { activity }\end{array}$ & $\begin{array}{l}\text { RAF1, SOS1, } \\
\text { RIN1, ABL2, } \\
\text { CAV1 }\end{array}$ & $\begin{array}{l}\text { Costello } \\
\text { syndrome }\end{array}$ & $\begin{array}{l}\text { oncogene, } \\
\text { MAPK } \\
\text { pathway }\end{array}$ & $\begin{array}{l}24768 \\
552\end{array}$ \\
\hline INTS6 & $\begin{array}{l}\text { Integrator } \\
\text { complex } \\
\text { subunit 6, } \\
\text { DICE1 }\end{array}$ & $\begin{array}{l}13 q 14 . \\
3\end{array}$ & $\begin{array}{l}\text { Component of } \\
\text { the Integrator } \\
\text { complex, } \\
\text { involved in the } \\
\text { small nuclear } \\
\text { RNAs } \\
\text { transcription } \\
\text { and } \\
\text { processing, } \\
\text { tumor } \\
\text { supressor }\end{array}$ & $\begin{array}{l}\text { UPF1, UPF2, } \\
\text { INTS1, INTS3, } \\
\text { INTS8 }\end{array}$ & $\begin{array}{l}\text { Autism, } \\
\text { susceptibility }\end{array}$ & lung cancer & $\begin{array}{l}26402 \\
605\end{array}$ \\
\hline KDM5B & $\begin{array}{l}\text { Lysine (K)- } \\
\text { Specific } \\
\text { Demethylase } \\
\text { 5B, JARID1B }\end{array}$ & $1 q 32.1$ & $\begin{array}{l}\text { Histone } \\
\text { demethylase } \\
\text { that } \\
\text { demethylates } \\
\text { K4 of histone } \\
\text { H3 }\end{array}$ & $\begin{array}{l}\text { ARID1B, RB1, } \\
\text { HDAC1, PAX9 }\end{array}$ & $\begin{array}{l}\text { Autism, } \\
\text { susceptibility }\end{array}$ & $\begin{array}{l}\text { Retinoblast } \\
\text { oma, } \\
\text { chromatin } \\
\text { regulation }\end{array}$ & $\begin{array}{l}25363 \\
768\end{array}$ \\
\hline KDM6B & $\begin{array}{l}\text { Lysine (K)- } \\
\text { Specific } \\
\text { Demethylase } \\
\text { 6B, JMJD3 }\end{array}$ & $\begin{array}{l}17 p 13 . \\
1\end{array}$ & $\begin{array}{l}\text { Histone } \\
\text { demethylase } \\
\text { that } \\
\text { specifically } \\
\text { demethylates } \\
\text { K27 of histone } \\
\text { H3 }\end{array}$ & $\begin{array}{l}\text { ESR1, } \\
\text { CSNK2B, } \\
\text { HIST1H3D }\end{array}$ & $\begin{array}{l}\text { Autism, } \\
\text { susceptibility }\end{array}$ & $\begin{array}{l}\text { Chromatin } \\
\text { regulation }\end{array}$ & $\begin{array}{l}25363 \\
768\end{array}$ \\
\hline KMT2C & \multicolumn{2}{|c|}{$\begin{array}{l}\text { Lysine (K)-Specific } \\
\text { Methyltransferase 2C, } \\
\text { MLL3 }\end{array}$} & $\begin{array}{l}\text { Histone } \\
\text { methyltransfer } \\
\text { ase that } \\
\text { methylates K4 } \\
\text { of histone H3 }\end{array}$ & $\begin{array}{l}\text { NCOA6, } \\
\text { ASCL2, } \\
\text { ASH2L, AK1, } \\
\text { TSC22D1 }\end{array}$ & $\begin{array}{l}\text { Autism, } \\
\text { susceptibility }\end{array}$ & $\begin{array}{l}\text { Lysine } \\
\text { degradation }\end{array}$ & $\begin{array}{l}26402 \\
605\end{array}$ \\
\hline KMT2D & MLL2 & $\begin{array}{l}12 \mathrm{q} 13 . \\
12\end{array}$ & $\begin{array}{l}\text { Histone } \\
\text { methylatransf } \\
\text { erase of K4me }\end{array}$ & $\begin{array}{l}\text { ESR1, PAXIPI, } \\
\text { RBBP5, } \\
\text { SMAD1, } \\
\text { SMAD9 }\end{array}$ & $\begin{array}{l}\text { Kabuki } \\
\text { syndrome }\end{array}$ & $\begin{array}{l}\text { Lysine } \\
\text { degradation }\end{array}$ & $\begin{array}{l}25891 \\
009\end{array}$ \\
\hline$M E C P 2$ & $\begin{array}{l}\text { Methyl CpG } \\
\text { binding }\end{array}$ & $\mathrm{Xq} 28$ & $\begin{array}{l}\text { chromosomal } \\
\text { protein and }\end{array}$ & $\begin{array}{l}\text { SIN3A, } \\
\text { SMARCA2, }\end{array}$ & Rett syndrome & Chromatin & 24779 \\
\hline
\end{tabular}




\begin{tabular}{|c|c|c|c|c|c|c|c|}
\hline & $\begin{array}{l}\text { protein 2, } \\
\text { AUTSX3 }\end{array}$ & & $\begin{array}{l}\text { transcriptional } \\
\text { regulator that } \\
\text { binds to } \\
\text { methylated } \\
\text { DNA }\end{array}$ & ATRX & & regulation & 060 \\
\hline MET & $\begin{array}{l}\text { AUTS9, HGFR, } \\
\text { c-Met }\end{array}$ & $7 q 31$ & $\begin{array}{l}\text { Receptor } \\
\text { tyrosine } \\
\text { kinase that } \\
\text { transduces } \\
\text { signals from } \\
\text { ECM by } \\
\text { binding HGF, } \\
\text { activates RAS- } \\
\text { ERK, AKT, or } \\
\text { PLC pathways }\end{array}$ & $\begin{array}{l}\text { HGF, CBL, } \\
\text { GRB2, UBC, } \\
\text { PTPN1 }\end{array}$ & $\begin{array}{l}\text { Autism, } \\
\text { association }\end{array}$ & $\begin{array}{l}\text { Hereditary } \\
\text { papillary } \\
\text { renal } \\
\text { carcinoma } \\
\text { (RCCP), } \\
\text { glioma, }\end{array}$ & $\begin{array}{l}19548 \\
256\end{array}$ \\
\hline MIB1 & $\begin{array}{l}\text { Mindbomb E3 } \\
\text { Ubiquitin } \\
\text { Protein Ligase } \\
1\end{array}$ & $\begin{array}{l}18 q 11 . \\
2\end{array}$ & $\begin{array}{l}\text { E3 ubiquitin- } \\
\text { protein ligase } \\
\text { that mediates } \\
\text { ubiquitination } \\
\text { of Delta } \\
\text { receptors, } \\
\text { which act as } \\
\text { ligands of } \\
\text { Notch proteins }\end{array}$ & $\begin{array}{l}\text { NOTCH1, } \\
\text { UBC, UBE2N, } \\
\text { DAPK1 }\end{array}$ & $\begin{array}{l}\text { Autism, } \\
\text { susceptibility }\end{array}$ & $\begin{array}{l}\text { Notch } \\
\text { signaling }\end{array}$ & $\begin{array}{l}26402 \\
605\end{array}$ \\
\hline NF1 & $\begin{array}{l}\text { Neurofibromin } \\
1 \text {, NFNS }\end{array}$ & $\begin{array}{l}17 q 11 . \\
2\end{array}$ & $\begin{array}{l}\text { Negative } \\
\text { regulator of } \\
\text { RAS signal } \\
\text { pathway }\end{array}$ & $\begin{array}{l}\text { GADD45A, } \\
\text { SMARCC1, } \\
\text { SMARCD1, } \\
\text { GTF2A1 }\end{array}$ & $\begin{array}{l}\text { Neurofibromato } \\
\text { sis, type } 1\end{array}$ & $\begin{array}{l}\text { Leukemia, } \\
\text { juvenile } \\
\text { myelomono } \\
\text { cytic } \\
\text { (JMML), } \\
\text { Ras, MAPK } \\
\text { pathways }\end{array}$ & $\begin{array}{l}24768 \\
552\end{array}$ \\
\hline NIPBL & $\begin{array}{l}\text { Nipped-B } \\
\text { Homolog } \\
\text { (Drosophila), } \\
\text { CDLS1 }\end{array}$ & $5 p 13.2$ & $\begin{array}{l}\text { cohesion } \\
\text { protein that } \\
\text { facilitates } \\
\text { enhancer- } \\
\text { promoter } \\
\text { interactions in } \\
\text { Drosophila }\end{array}$ & $\begin{array}{l}\text { SMC3, } \\
\text { HDAC1, } \\
\text { HDAC2, } \\
\text { ATAD5 }\end{array}$ & $\begin{array}{l}\text { Cornelia de } \\
\text { Lange syndrome } \\
1\end{array}$ & $\begin{array}{l}\text { colorectal } \\
\text { and gastric } \\
\text { cancer }\end{array}$ & $\begin{array}{l}24768 \\
552\end{array}$ \\
\hline PAX5 & $\begin{array}{l}\text { Paired Box 5, } \\
\text { ALL3, BSAP }\end{array}$ & $9 p 13.2$ & $\begin{array}{l}\text { Paired box } \\
\text { transcription } \\
\text { factor involved } \\
\text { in B cell } \\
\text { development, } \\
\text { neural } \\
\text { development, } \\
\text { spermatogene } \\
\text { sis; recurrent } \\
\text { translocations }\end{array}$ & $\begin{array}{l}\text { EP300, } \\
\text { CEBBP, ETS1, } \\
\text { TBP, EBF1 }\end{array}$ & $\begin{array}{l}\text { Autism, } \\
\text { susceptibility }\end{array}$ & $\begin{array}{l}\text { Leukemia, } \\
\text { acute } \\
\text { lymphoblast } \\
\text { ic, } \\
\text { susceptibilit } \\
\text { y (ALL3), } \\
\text { WNT } \\
\text { pathway }\end{array}$ & $\begin{array}{l}25418 \\
537\end{array}$ \\
\hline
\end{tabular}




\begin{tabular}{|c|c|c|c|c|c|c|c|}
\hline & & & in lymphoma & & & & \\
\hline PHF2 & $\begin{array}{l}\text { PHD Finger } \\
\text { Protein } 2\end{array}$ & $\begin{array}{l}9 q 22.3 \\
1\end{array}$ & $\begin{array}{l}\text { Lysine histone } \\
\text { demethylase } \\
\text { that is } \\
\text { recruited to } \\
\text { trimethylated } \\
\text { Lys-4 of } \\
\text { histone H3 } \\
\text { (H3K4me3) at } \\
\text { rDNA } \\
\text { promoters and } \\
\text { promotes } \\
\text { expression of } \\
\text { rDNA }\end{array}$ & $\begin{array}{l}\text { TP53, RBBP7, } \\
\text { SUZ12, EZH2 }\end{array}$ & $\begin{array}{l}\text { Autism, } \\
\text { susceptibility }\end{array}$ & $\begin{array}{l}\text { Chromatin } \\
\text { regulation }\end{array}$ & $\begin{array}{l}26402 \\
605\end{array}$ \\
\hline PTEN & MMAC1 & $\begin{array}{l}10 q 23 . \\
3\end{array}$ & $\begin{array}{l}\text { tumor } \\
\text { suppressor, } \\
\text { dual- } \\
\text { specificity } \\
\text { protein } \\
\text { phosphatase }\end{array}$ & $\begin{array}{l}\text { NEDD4, } \\
\text { AKT1, PTK2, } \\
\text { UBC, } \\
\text { SLC9A3R1 }\end{array}$ & $\begin{array}{l}\text { Macrocephaly/a } \\
\text { utism syndrome }\end{array}$ & $\begin{array}{l}\text { Cowden } \\
\text { syndrome, } \\
\text { glioblastom } \\
\text { a, mTOR } \\
\text { pathway, } \\
\text { recombinati } \\
\text { onal DNA } \\
\text { repair }\end{array}$ & $\begin{array}{l}24768 \\
552\end{array}$ \\
\hline PTK7 & \multicolumn{2}{|c|}{$\begin{array}{l}\text { Protein Tyrosine Kinase } \\
7 \text { (Inactive) }\end{array}$} & $\begin{array}{l}\text { Inactive } \\
\text { tyrosine } \\
\text { kinase } \\
\text { involved } \\
\text { incononical } \\
\text { and non- } \\
\text { cononical Wnt } \\
\text { signaling } \\
\text { pathways, } \\
\text { function in cell } \\
\text { adhesion, cell } \\
\text { migration, cell } \\
\text { polarity, } \\
\text { proliferation, } \\
\text { actin } \\
\text { cytoskeleton } \\
\text { reorganization } \\
\text { and apoptosis }\end{array}$ & $\begin{array}{l}\text { DVL1, DVL2, } \\
\text { DVL3, } \\
\text { CTNNB1, } \\
\text { WNT9B }\end{array}$ & $\begin{array}{l}\text { Autism, } \\
\text { susceptibility }\end{array}$ & $\begin{array}{l}\text { WNT and } \\
\text { AKT } \\
\text { signaling }\end{array}$ & $\begin{array}{l}26402 \\
605\end{array}$ \\
\hline$S M C 1 A$ & $\begin{array}{l}\text { Structural } \\
\text { Maintenance } \\
\text { Of } \\
\text { Chromosomes } \\
1 \mathrm{~A}\end{array}$ & $\begin{array}{l}\text { Xp11.2 } \\
2\end{array}$ & $\begin{array}{l}\text { chromosome } \\
\text { cohesion } \\
\text { during cell } \\
\text { cycle and DNA } \\
\text { repair }\end{array}$ & $\begin{array}{l}\text { SMC3, } \\
\text { RAD21, } \\
\text { STAG2, } \\
\text { SMC2, SSU72 }\end{array}$ & $\begin{array}{l}\text { Cornelia de } \\
\text { Lange syndrome } \\
2\end{array}$ & $\begin{array}{l}\text { genome } \\
\text { maintenanc } \\
\text { e, colorectal } \\
\text { cancer }\end{array}$ & $\begin{array}{l}24768 \\
552\end{array}$ \\
\hline
\end{tabular}




\begin{tabular}{|c|c|c|c|c|c|c|c|}
\hline SMC2 & $\begin{array}{l}\text { Structural } \\
\text { Maintenance } \\
\text { Of } \\
\text { Chromosomes } \\
2\end{array}$ & $9 q 31.1$ & $\begin{array}{l}\text { critical for } \\
\text { mitotic } \\
\text { chromosome } \\
\text { condensation } \\
\text { and for DNA } \\
\text { repair }\end{array}$ & $\begin{array}{l}\text { SMC1A, } \\
\text { SMC4, } \\
\text { NCAPH, } \\
\text { NCAPH2, } \\
\text { NCAPD2 }\end{array}$ & $\begin{array}{l}\text { High confidence } \\
\text { ASD candidate } \\
\text { gene }\end{array}$ & $\begin{array}{l}\text { Genome } \\
\text { maintenanc } \\
\mathrm{e}\end{array}$ & $\begin{array}{l}24768 \\
552\end{array}$ \\
\hline $\begin{array}{l}\text { SUV420 } \\
\text { H1 }\end{array}$ & $\begin{array}{l}\text { Lysine } \mathrm{N} \text { - } \\
\text { Methyltransfer } \\
\text { ase 5B, KMT5B }\end{array}$ & $\begin{array}{l}11 q 13 . \\
2\end{array}$ & $\begin{array}{l}\text { Histone } \\
\text { methyltransfer } \\
\text { ase that } \\
\text { specifically } \\
\text { trimethylates } \\
\text { K20 of histone } \\
\text { H4 }\end{array}$ & $\begin{array}{l}\text { TP53BP1, } \\
\text { NCOA2, } \\
\text { YWHAQ }\end{array}$ & $\begin{array}{l}\text { Autism, } \\
\text { susceptibility }\end{array}$ & $\begin{array}{l}\text { Lysine } \\
\text { degradation }\end{array}$ & $\begin{array}{l}26402 \\
605\end{array}$ \\
\hline $\begin{array}{l}\text { TBL1XR } \\
1\end{array}$ & $\begin{array}{l}\text { Transducin } \\
\text { (Beta)-Like } 1 \text { X- } \\
\text { linked } \\
\text { Receptor 1, } \\
\text { TBLR1, IRA1 }\end{array}$ & $\begin{array}{l}3 q 26.3 \\
2\end{array}$ & $\begin{array}{l}\text { F-box-like } \\
\text { protein } \\
\text { recruits } \\
\text { ubiquitin/19S } \\
\text { proteosome } \\
\text { complex to } \\
\text { nuclear } \\
\text { hormone } \\
\text { receptors, } \\
\text { degradation of } \\
\mathrm{N} \text {-Cor for } \\
\text { transcriptional } \\
\text { activation }\end{array}$ & $\begin{array}{l}\text { TBL1X, } \\
\text { HDAC3, } \\
\text { NCOR1, } \\
\text { THRB, } \\
\text { CACNA1C, } \\
\text { CACNA1E }\end{array}$ & $\begin{array}{l}\text { Autism, } \\
\text { susceptibility }\end{array}$ & $\begin{array}{l}\text { NOTCH1, } \\
\text { PPARalpha } \\
\text { metabolism }\end{array}$ & $\begin{array}{l}26069 \\
883\end{array}$ \\
\hline TCF7L2 & $\begin{array}{l}\text { T-Cell-Specific } \\
\text { Transcription } \\
\text { Factor } 4\end{array}$ & $\begin{array}{l}10 q 25 . \\
2\end{array}$ & $\begin{array}{l}\text { High mobility } \\
\text { group (HMG) } \\
\text { box-containing } \\
\text { transcription } \\
\text { factor that } \\
\text { plays a key } \\
\text { role in the } \\
\text { Wnt signaling } \\
\text { pathway }\end{array}$ & $\begin{array}{l}\text { TCF7, } \\
\text { CTNNB1, } \\
\text { RUVBL2 }\end{array}$ & $\begin{array}{l}\text { Autism, } \\
\text { susceptibility }\end{array}$ & $\begin{array}{l}\text { WNT } \\
\text { signalling }\end{array}$ & $\begin{array}{l}25363 \\
768\end{array}$ \\
\hline $\begin{array}{l}\text { TNRC6 } \\
B\end{array}$ & $\begin{array}{l}\text { Trinucleotide } \\
\text { Repeat } \\
\text { Containing 6B }\end{array}$ & $\begin{array}{l}22 q 13 . \\
1\end{array}$ & $\begin{array}{l}\text { Plays a role in } \\
\text { RNA-mediated } \\
\text { gene silencing } \\
\text { by both micro- } \\
\text { RNAs } \\
\text { (miRNAs) and } \\
\text { short } \\
\text { interfering } \\
\text { RNAs (siRNAs) }\end{array}$ & $\begin{array}{l}\text { TP53, AGO1, } \\
\text { CDK4, EIF2C1 }\end{array}$ & $\begin{array}{l}\text { Autism, } \\
\text { susceptibility }\end{array}$ & PI-3K & $\begin{array}{l}25363 \\
768\end{array}$ \\
\hline TRIO & $\begin{array}{l}\text { Trio Rho } \\
\text { Guanine } \\
\text { Nucleotide } \\
\text { Exchange }\end{array}$ & $5 p 15.2$ & $\begin{array}{l}\text { Promotes the } \\
\text { exchange of } \\
\text { GDP by GTP, } \\
\text { coordinates }\end{array}$ & $\begin{array}{l}\text { RAC1, RAC3, } \\
\text { HCRTR2, } \\
\text { DISC1, CDC5L }\end{array}$ & $\begin{array}{l}\text { Autism, } \\
\text { susceptibility }\end{array}$ & $\begin{array}{l}\text { NOTCH, Rho } \\
\text { GTPase }\end{array}$ & $\begin{array}{l}26402 \\
605\end{array}$ \\
\hline
\end{tabular}




\begin{tabular}{|c|c|c|c|c|c|c|c|}
\hline & Factor & & $\begin{array}{l}\text { cell-matrix and } \\
\text { cytoskeletal } \\
\text { rearrangemen } \\
\text { ts necessary } \\
\text { for cell } \\
\text { migration and } \\
\text { cell growth }\end{array}$ & & & & \\
\hline TRIP12 & $\begin{array}{l}\text { Thyroid } \\
\text { hormone } \\
\text { receptor } \\
\text { interating } \\
\text { protein, E3 } \\
\text { Ubiquitin- } \\
\text { Protein Ligase } \\
\text { For Arf }\end{array}$ & $2 q 36.3$ & $\begin{array}{l}\text { E3 ubiquitin- } \\
\text { protein ligase } \\
\text { involved in } \\
\text { ubiquitin } \\
\text { fusion } \\
\text { degradation } \\
\text { pathway, } \\
\text { suppresses } \\
\text { spreading of } \\
\text { Ub-chromatin } \\
\text { at damaged } \\
\text { chromosomes }\end{array}$ & $\begin{array}{l}\text { MYC, TRADD, } \\
\text { SMARCC1, } \\
\text { CDKN2A, } \\
\text { SMARCE1, } \\
\text { THRB, } \\
\text { PSMC5, } \\
\text { TMEFF2 }\end{array}$ & $\begin{array}{l}\text { Autism, } \\
\text { susceptibility }\end{array}$ & $\begin{array}{l}\text { Class I MHC } \\
\mathrm{Ag} \\
\text { presentatio } \\
\mathrm{n} \text { and } \\
\text { processing }\end{array}$ & $\begin{array}{l}25418 \\
537\end{array}$ \\
\hline TSC1 & $\begin{array}{l}\text { Tuberous } \\
\text { Sclerosis 1, } \\
\text { LAM }\end{array}$ & $\begin{array}{l}9 q 34.1 \\
3\end{array}$ & $\begin{array}{l}\text { Negative } \\
\text { regulation of } \\
\text { mTORC1 } \\
\text { signalling }\end{array}$ & $\begin{array}{l}\text { TSC2, } \\
\text { MAPK1, } \\
\text { RHEB, AKT1, } \\
\text { IKBKB }\end{array}$ & $\begin{array}{l}\text { Tuberous } \\
\text { sclerosis }\end{array}$ & $\begin{array}{l}\text { MTOR, AKT } \\
\text { pathway }\end{array}$ & $\begin{array}{l}24768 \\
552\end{array}$ \\
\hline TSC2 & $\begin{array}{l}\text { Tuberous } \\
\text { Sclerosis 2, } \\
\text { TSC4, LAM }\end{array}$ & $\begin{array}{l}16 \mathrm{p} 13 . \\
3\end{array}$ & $\begin{array}{l}\text { Negative } \\
\text { regulation of } \\
\text { mTORC1 } \\
\text { signalling }\end{array}$ & $\begin{array}{l}\text { TSC1, RHEB, } \\
\text { YWHAZ, } \\
\text { YWAB }\end{array}$ & $\begin{array}{l}\text { Tuberous } \\
\text { sclerosis }\end{array}$ & $\begin{array}{l}\text { MTOR, AKT } \\
\text { pathway }\end{array}$ & $\begin{array}{l}24768 \\
552\end{array}$ \\
\hline UBE3A & $\begin{array}{l}\text { E6AP } \\
\text { Ubiquitin- } \\
\text { Protein Ligase, } \\
\text { ANCR }\end{array}$ & $\begin{array}{l}15 q 11 . \\
2\end{array}$ & $\begin{array}{l}\text { E3 ubiquitin- } \\
\text { protein ligase, } \\
\text { cofactor for } \\
\text { nuclear } \\
\text { hormone } \\
\text { receptors, } \\
\text { maternal } \\
\text { mutations } \\
\text { cause } \\
\text { Angelman } \\
\text { syndrome, } \\
\text { imprinted in } \\
\text { brain, in } \\
\text { cervical cancer } \\
\text { degrades p53 } \\
\text { in presence of } \\
\text { E6 }\end{array}$ & $\begin{array}{l}\text { RAD23A, } \\
\text { HERC2, } \\
\text { RING1B, } \\
\text { ESR1, RARA }\end{array}$ & $\begin{array}{l}\text { Angelman } \\
\text { syndrome (del), } \\
\text { Dup15q } \\
\text { syndrome (dup) }\end{array}$ & $\begin{array}{l}\text { Class I MHC } \\
\text { Ag } \\
\text { presentatio } \\
\mathrm{n} \text { and } \\
\text { processing, } \\
\text { PEDF, } \\
\text { estrogen }\end{array}$ & $\begin{array}{l}24779 \\
060\end{array}$ \\
\hline WAC & $\begin{array}{l}\text { WW Domain } \\
\text { Containing } \\
\text { Adaptor With }\end{array}$ & $\begin{array}{l}10 p 12 . \\
1\end{array}$ & $\begin{array}{l}\text { Acts as a linker } \\
\text { between gene } \\
\text { transcription } \\
\text { and histone }\end{array}$ & $\begin{array}{l}\text { UBC, } \\
\text { UBQLN4, } \\
\text { POL2R2A }\end{array}$ & $\begin{array}{l}\text { Autism, } \\
\text { susceptibility }\end{array}$ & $\begin{array}{l}\text { chromatin } \\
\text { regulation }\end{array}$ & $\begin{array}{l}26402 \\
605\end{array}$ \\
\hline
\end{tabular}




\begin{tabular}{|l|l|l|l|l|l|l|l|}
\hline Coiled-Coil & $\begin{array}{l}\text { H2B } \\
\text { monoubiquitin } \\
\text { ation at K120 }\end{array}$ & & & & \\
\hline
\end{tabular}

*Genes summarized in Table 1 were identified as autism risk genes from publications in the cited references identified by PIMD numbers in the far right column. Information describing each gene was assembled from sources compiled within GeneCards and OMIM databases.

\section{Acknowledgments}

Supported by Autism Speaks Targeted Award \#8703 (JNC), NINDS 1R01NS085709-01 (JNC), NICHD U54 HD079125 (JNC), NCI CA92276 (WDH), NCI CA154920 (WDH), NIHGMS GM58015 (WDH), DOD W81XWH-14-1-0435 (WDH), NINDS R01NS081913 and R01NS076263 (JML), NIEHS R01ES021707 and 2P01ES011269 (JML).

\section{References}

1 Lord, C. and Bishop, S.L. (2015) Recent advances in autism research as reflected in DSM-5 criteria for autism spectrum disorder. Ann. Rev. Clin. Psychol. 11, 53-70

2 Bourgeron, T. (2015) From the genetic architecture to synaptic plasticity in autism spectrum disorder. Nature Reviews 16, 551-563

3 Iossifov, I., et al. (2014) The contribution of de novo coding mutations to autism spectrum disorder. Nature 515, 216-221

4 De Rubeis, S., et al. (2014) Synaptic, transcriptional and chromatin genes disrupted in autism. Nature 515, 209-215

5 Geschwind, D.H. and State, M.W. (2015) Gene hunting in autism spectrum disorder: on the path to precision medicine. Lancet Neurol. S1474-4422(15)00044-7 
6 Martincorena, I. and Campbell, P.J. (2015) Somatic mutation in cancer and normal cells. Science 349, 1483-1489

7 Zhou, J. and Parada, L.F. (2012) PTEN signaling in autism spectrum disorders. Curr. Opin. Neurobiol. 22, 873-879

8 Pinto, D., et al. (2014) Convergence of genes and cellular pathways dysregulated in autism spectrum disorders. Am. J. Hum. Genet. 94, 677-694

9 Crespi, B. (2011) Autism and cancer risk. Autism Res. 4, 302-310

10 LaSalle, J.M., et al. (2015) Epigenetic regulation of UBE3A and roles in human neurodevelopmental disorders. Epigenomics 7, 1213-1218

11 Aguilera, A. and Garcia-Muse, T. (2013) Causes of genome instability. Annu. Rev. Genet. 47, $1-32$

12 Chiang, H.-L., Liu, et al. (2015) Risk of cancer in children, adolescents, and young adults with autistic disorder. J. Pedriatics 166, 418-423

13 Kazdoba, T.M., et al. (2015) Behavioral phenotypes of genetic mouse models of autism. Genes Brain Behav. doi: 10.1111/gbb.12256. PMID: 26403076 